\title{
Incorporating stimulus-responsive character into filamentous virus assemblies
}

\author{
Harry Bermudez,* Adam P. Hathorne \\ ${ }_{5}$ DOI: 10.1039/b000000x [DO NOT ALTER/DELETE THIS TEXT]
}

Controlling interactions between building blocks, in either guided or selfassemblies, is becoming increasingly important for creation of functional materials. We have focused our attention on the well-known model assembly, the filamentous bacteriophage, where our strategy is to

${ }_{10}$ selectively alter surface features by focusing on spatially distinct capsid proteins. Towards introducing stimulus-responsive behavior in these flexible, rod-like particles, we have introduced elastin-like polypeptide (ELP) motifs of isoleucine and tyrosine "guest" residues by recombinant DNA methods. Our hypothesis is that modification of the major coat ${ }_{15}$ capsid protein would be greatly amplified by the 2700 copies per particle. Characterization of ELP-phage particles was carried out by microbiological assays, zeta potential, dynamic light scattering, and calorimetry. Bacteria producing ELP-phage particles grow more slowly and surprisingly, ELPmodified phages display a significant reduction in viral infectivity. For the ${ }_{20}$ lengths of ELP inserts studied, modified phages do not aggregate from solution as monitored by DLS. However, the hydrodynamic size of the phages depends on the details of the ELP motif. Zeta potential measurements reveal the particles are electrostatically stabilized, and this contributes in part to the energetic barrier against aggregation. Preliminary ${ }_{25}$ calorimetric data indicate subtle thermal transitions in the range $35-40^{\circ} \mathrm{C}$, suggesting that the ELP motif may collapse without triggering macroscopic aggregation. The results are consistent with the classical picture of critical solution phenomena at low concentrations, where to drive phase separation, solvent quality must be increasingly poor. Apart from being model systems 30 to study basic questions of self-assembly, extending the these modular systems is likely to result in improved understanding and control over selfassembly in various applications.

\section{Introduction}

Self-assembly in solution relies not only on thermodynamic and kinetic factors but 35 on the directionality of interactions. It is this directionality that distinguishes useful "assembly" from undesirable "aggregation". Well-known assemblies such as micelles and vesicles are symmetric largely due to the weak directional nature of hydrogen bonding (hydrophobic effect) that drives their formation in water.

Department of Polymer Science \& Engineering, University of Massachusetts, Amherst MA 01003 USA. Fax: +1 413545 0082; Tel: +1 413577 1413; E-mail: bermudez@polysci.umass.edu

[journal], [year], [vol], 00-00 | 1 
Additional types of long-range interactions lead to more complex structures, 40 accounting for the vast difference between an idealized collapsed polymer globule and a folded functional protein. Yet in spite of that difference, theoretical developments such as lattice models have greatly improved our understanding of protein folding and stability. ${ }^{1}$ It becomes clear that objects with high degrees of symmetry have limited functionality and thus there is an increased effort towards the ${ }_{45}$ creation of asymmetric assemblies. ${ }^{2-4}$

Asymmetry is relevant at multiple length scales in biology, from the molecularlevel orientation of transmembrane proteins to the tissue level where it is essential to development. To the materials scientist, the tools of protein engineering combined with relatively simple biological model systems provide opportunities to establish

${ }_{50}$ design criteria for creating new asymmetric assemblies. One such widely known model system is the filamentous bacteriophage. ${ }^{5}$ Indeed, phages as a family provided the foundation of molecular biology 6,7 and their numerous members are now finding diverse applications. ${ }^{8-12}$ The protein capsid of M13 filamentous bacteriophage consists of only five types of structural proteins, ${ }^{13}$ allowing the use of ${ }_{55}$ molecular biology to tailor these viruses with a degree of precision that is difficult to achieve with traditional inorganic particles. The major capsid protein, also known as pVIII, is present in approximately 2700 copies per phage and thus makes up the majority of the surface (Figure 1). The minor coat protein pIII has a far smaller copy number of only five, yet it plays a key role in viral infectivity. This natural ${ }_{60}$ decoupling of spatial location and function inherent in the viral capsid suggests an approach to alter bacteriophage properties by manipulating specific capsid proteins (Figure 1).

(a)

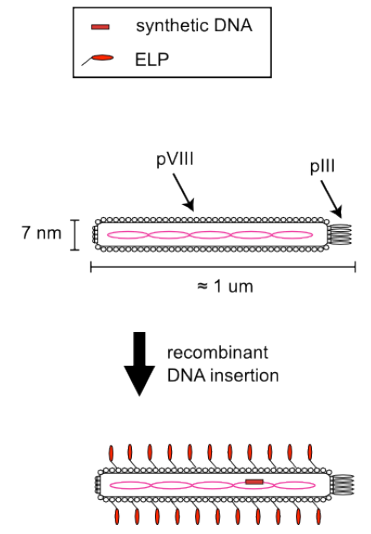

(b)

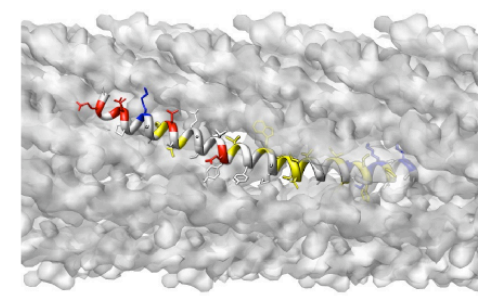

Fig. 1 (a) Schematic of filamentous bacteriophage structure, depicting the major (pVIII) and minor 65 (pIII) coat proteins; the remaining three types of structural proteins are not shown. Recombinant DNA insertion leads to displayed peptides on each copy of the corresponding capsid protein. (b) Section of a multimolecular model (PDB ID: 1IFJ) of the phage capsid showing alpha-helical nature of $\mathrm{pVIII}$ and the staggered orientation of the many $\mathrm{pVIII}$ proteins. One copy of $\mathrm{pVIII}$ is highlighted for clarity.

70 Apart from their role in virology, phages can be used to explore fundamental questions of self-assembly in the context of the capsid. Such viruses can also be thought of as tunable colloids or nanoscale scaffolds. ${ }^{14,15}$ Control over placement of

2 | [journal], [year], [vol], 00-00 
reactive groups on virus particles such as phages has been clearly demonstrated, ${ }^{16,17}$ but responsiveness to environmental stimuli is still lacking. As a step in this 75 direction we have used recombinant DNA techniques to display elastin-like polypeptides (ELPs) on the surface of filamentous bacteriophage particles. The thermoresponsive character of elastin-like polypeptides varies with the length and composition of the genetically encoded monomeric unit: (VPGXG) $)_{n}$ where $\mathrm{X}$ denotes any amino acid. ${ }^{18}$ The molecular description of the ELP transition is still

80 unclear, but it is generally believed to be explained by increasingly poor hydration of the polypeptide, leading to intramolecular beta-type interactions. ${ }^{19,} 20$ Nevertheless, ELPs have found numerous applications exploiting their switching behavior, ranging from the macroscopic to the single-molecule level. ${ }^{21-25}$ Due to the inverse dependence of transition temperature $T_{t}$ with chain length, ${ }^{26}$ we have

85 focused our attention on short segments with $n=1,3,6$ where hydrophobic residues are placed in the "guest" position. In principle, by varying the guest residue and/or chain length, the responsive behavior could be modulated with a high degree of control.

\section{Experimental}

\section{${ }_{90}$ ELP fusion construction}

Synthetic oligonucleotides containing the ELP sequence were designed and purchased from Integrated DNA Technologies. To form appropriate overhangs for ligation, four to six oligos were annealed from $95^{\circ} \mathrm{C}$ to $15^{\circ} \mathrm{C}$ at a rate of $-1{ }^{\circ} \mathrm{C} / \mathrm{min}$ to generate the double-stranded insert. The $5^{\prime}$-ends of the insert were subsequently ${ }_{95}$ phosphorylated with $\mathrm{T} 4$ polynucleotide kinase. The M13KO7 phage vector (Amersham) was digested at two sites with the restriction enzyme BbvCI, gel purified and extracted (Qiagen). Vector and insert were ligated with T4 DNA ligase at a 3:1 insert:vector ratio. Subsequent transformation of the plasmid into either DH5a (Invitrogen) or TG1 (Biochain) E. coli competent cells was performed 100 according to standard techniques. Positive selection for transformants was acheived by use of kanamycin resistance. All enzymes were purchased from New England Biolabs.

To screen clones for ELP insertion, plasmid DNA was isolated from single colony cultures using a Qiaprep Spin Miniprep Kit (Qiagen). Plasmid concentrations were 105 measured using a Nanodrop ND-1000 spectrophotometer. PCR screening primers, flanking and straddling the insert region, were designed and purchased (IDTDNA) and used to amplify desired target regions. PCR products were visualized on a $1.2 \%$ agarose gel to identify positive inserts. Upon PCR confirmation of the insert, plasmid DNA was sequenced (GeneWiz) with the upstream primer 5' 110 AGGTtGgtGCCTTCGTAG-3' to ensure no errors were present.

\section{Phage isolation}

Large-scale cultures of 200-250 mL 2xYT media (Teknova) with $15 \mathrm{ug} / \mathrm{mL}$ kanamycin were grown from single colonies of each respective ELP clone. After centrifuging the cultures for 30 minutes at $3000 \mathrm{~g}$ to remove bacterial cells, the 115 supernatant was collected and 0.15 vol $\mathrm{PEG}-8000 / \mathrm{NaCl}$ solution $(18 \% \mathrm{w} / \mathrm{v}, 2 \mathrm{M})$ added. Solutions were gently mixed and incubated at $4{ }^{\circ} \mathrm{C}$ for $3 \mathrm{hr}$, followed by centrifugation at $3100 \mathrm{~g}$ for $80 \mathrm{~min}$ to pellet the phages. The supernatant was removed and 20-30 mL of 1x PBS added to resuspend the phage pellet. The solution

[journal], [year], [vol], 00-00 | 3 
was centrifuged at $10,000 \mathrm{~g}$ for $10 \mathrm{~min}$ to clear the supernatant and a second 120 precipitation was performed by adding 0.15 vol PEG- $8000 / \mathrm{NaCl}$ to the supernatant, followed by incubation at $4^{\circ} \mathrm{C}$ overnight. The phages were re-pelleted by centrifugation at $20,000 \mathrm{~g}$ for $40 \mathrm{~min}$. After removal of the supernatant, the phage pellet was resuspended in $5 \mathrm{~mL}$ of $1 \mathrm{x}$ PBS by gently shaking at room temperature. Solutions were spun one final time at $10,000 \mathrm{~g}$ for $10 \mathrm{~min}$ to remove any remaining ${ }_{125}$ debris and the supernatant stored at $4{ }^{\circ} \mathrm{C}$. Unless otherwise specified, reagents were purchased from Sigma-Aldrich and Fisher Scientific.

\section{Characterization}

Analysis of the ELP-phage behavior was performed using microbiological assays, dynamic light scattering, zeta potential, and calorimetry. Growth curves were 130 developed for bacteria harboring each ELP in order to determine whether the incorporation of the ELP into the pVIII protein had any impact on host growth rate. The optical density at $600 \mathrm{~nm}$ of bacterial cultures was monitored using a Nanodrop ND-1000 spectrophotometer. Time constants were calculated based on semi-log plots of absorbance versus time. Viral infectivity, also known titering, was 135 determined by mixing known dilutions of phage solutions with uninfected TG1 $E$. coli bacteria and incubating for $30 \mathrm{~min}$ at $37^{\circ} \mathrm{C}$. Spots of the mixture were subsequently applied onto LB agar plates with $40 \mathrm{ug} / \mathrm{mL}$ kanamycin and incubated overnight to allow colony formation.

A Nano-ZS Nanoseries Zetasizer (Malvern Instruments) was used to examine 140 hydrodynamic size and zeta potential. Approximately 400-500 uL of phage solutions were loaded into PMMA low volume disposable cuvettes. Temperature scans were conducted from $5^{\circ} \mathrm{C}$ to $75^{\circ} \mathrm{C}$ with an interval $10-15^{\circ} \mathrm{C}$ and a 2 min equilibration time. In order to determine if deprotonation of the tyrosine guest residue would result in a shift of the transition temperature, the $\mathrm{pH}$ of the phage solutions was adjusted using

${ }_{145}$ 0.1-1 M NaOH. Zeta potential measurements were conducted at $25^{\circ} \mathrm{C}$ using disposable capillary cuvettes (Malvern).

Calorimetric data was acquired with a TA Instruments Q1000 differential scanning calorimeter. Approximately $20-25 \mathrm{mg}$ of sample was loaded into a aluminum chamber and hermetically sealed. Samples were heated and cooled at a ${ }_{150}$ rate of $10^{\circ} \mathrm{C} / \mathrm{min}$ from $20-70^{\circ} \mathrm{C}$, using air as a reference.

\section{Results and Discussion}

\section{ELP fusion design}

Gene VIII, which encodes the major coat protein (pVIII), contains two BbvCI restriction sites. The first site cuts at DNA encoding for the alanine residue A1 at 155 the amino terminus of the mature protein, and the second site after A16. Thus to properly construct the ELP fusion required re-introducing approximately $50 \mathrm{bp}$ of DNA encoding residues 2-16 (see Table 1). In that process we performed silent mutations in the mentioned region to enhance the codon preference of E. coli: specifically, Pro (CCC to CCG), Ala (GCN to GCT), Phe (TTT to TTC).

160 The position of the first BbvCI site is additionally useful because of the proximity to the amino terminus of pVIII, and hence fusions at that position are likely to be solvent-accessible rather than buried in the capsid interior. Indeed, it is known that single cysteines on phage capsids display sequence/position dependent reactivity. ${ }^{27}$ Also important is preserving the ability of the modified protein pVIII to properly

4 | [journal], [year], [vol], 00-00 
165 self-assemble in the bacterial cell membrane, a process still poorly understood ${ }^{13}$ but known to involve both electrostatic interactions near the carboxy terminus and hydrophobic residues in the center of pVIII.

Table 1 DNA and amino acid sequence of the phage major coat protein pVIII (residues 1-17):

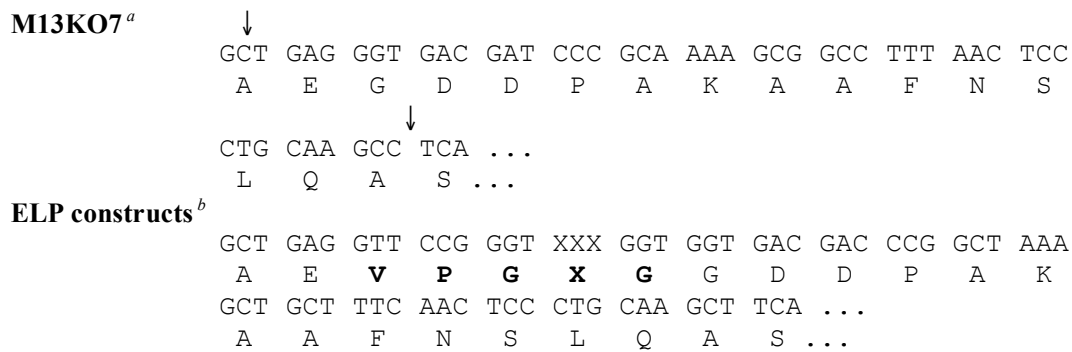

${ }^{a}$ Arrows denote BbvCI sites. ${ }^{b} \mathrm{X}$ is the "guest" residue in the ELP motif, in this study either tyrosine $170(\mathrm{Y})$ or isoleucine $(\mathrm{I})$.

The ELP clones are named according to the guest residue $X$ and number of repeats, $\mathrm{n}$ : for example $(\mathrm{VPGYG})_{3}$ is denoted as $\mathrm{Y}_{3}$. Because of the inverse dependence of transition temperature $T_{t}$ with chain length, ${ }^{26}$ increases in $T_{t}$ at short 175 chain lengths would be presumably offset by the low "asymptotic" $T_{t, \infty}$ at very long chain lengths $(n>150)$. Thus isoleucine and tyrosine were chosen as guest residues, having asymptotic $\mathrm{T}_{\mathrm{t}, \infty}$ of $10^{\circ} \mathrm{C}$ and $-55^{\circ} \mathrm{C}$, respectively. There has been limited investigation of relatively short ELP sequences, with a few notable exceptions. For example, Reiersen and coworkers ${ }^{28}$ used solid-phase peptide synthesis and circular 180 dichroism to examine short versions of the classical (VPGVG) $)_{n}$ motif where $n=1,3,5$. In contrast to the predictions of both Urry and Chilkoti, they found $T_{t}$ to range from $21-41^{\circ} \mathrm{C}$ at $\mathrm{pH}$ 7. This is a rather modest change given that the asymptotic $\mathrm{T}_{\mathrm{t}, \infty}$ for valine is $21^{\circ} \mathrm{C}$, and even more surprising when compared to the predicted ${ }^{26}$ $\mathrm{T}_{\mathrm{t}}=163^{\circ} \mathrm{C}$ for $\mathrm{n}=5$. It must be pointed out that such small ELPs might undergo 185 structural transitions yet not exhibit sufficient hydrophobicity to drive aggregation of the much larger particles in dilute solutions.

In our ELP-phage solutions, the ELP concentration does not exceed $5 \mathrm{uM}$, in spite of the high copy number of pVIII on phage particles. However, as the ELP is essentially "grafted" to each pVIII, the local concentration is effectively that of a 190 brush with a density of 2700 copies per particle surface area. Taking the brush length as the ELP contour length gives a minimum local concentration of $170 \mathrm{uM}$ for $\mathrm{n}=1$, down to $28 \mathrm{uM}$ for $\mathrm{n}=6$; a significant enhancement compared to the "bulk" concentration. Even so, at low concentrations and short chain lengths, there is still evidence for ELP functionality. For example, in two separate cases of ELP linkers 195 between chains of antibody fragments, thermoresponsive binding effects were observed with as little as a single repeat ELP unit. ${ }^{25,29}$

Apart from the thermoresponsive behavior of ELP motifs, certain amino acids have inherent characteristics which might be additionally exploited. For example, tyrosine has a $\mathrm{pK}_{\mathrm{a}}$ of 10.1 , and when deprotonated has an asymptotic $\mathrm{T}_{\mathrm{t}, \infty}=120^{\circ} \mathrm{C}$; 200 thus for short ELP sequences, $\mathrm{T}_{\mathrm{t}}$ should be elevated even higher, making it effectively inaccessible. Thus by changing the $\mathrm{pH}$ of the phage environment, the tyrosine ELP clones $\left(\mathrm{Y}_{1}, \mathrm{Y}_{3}, \mathrm{Y}_{6}\right)$ should exhibit a $\mathrm{pH}$-dependent transition above the $\mathrm{pK}_{\mathrm{a}}$.

[journal], [year], [vol], 00-00 | 5 


\section{Growth of infected $E$. coli and infectivity of ELP-phages}

${ }_{205}$ One of our concerns was that upon synthesis, the hydrophobic ELP-modified pVIII proteins would cause intracellular aggregation, leading to growth inhibition or even death of the host bacteria before significant amounts of virus could be produced. To check for this possibility, we monitored the optical density at $600 \mathrm{~nm}$ through the exponential phase for all constructs. The time constants measured are all either 210 slower or equal in growth compared to its $\mathrm{M} 13 \mathrm{KO} 7$ counterpart (Figure 2a). This result suggests the transition temperature is near or below $37^{\circ} \mathrm{C}$, or that intracellular levels of the ELP-modified pVIII begins to accumulate to inhibitory concentrations, although this would be mitigated by active secretion of virus particles. In spite of the slower growth, virus particles are still produced in comparable numbers by the

215 infected bacteria (data not shown). It is well-known that virus production diverts energy and resources from the natural growth of the bacteria, and it may be even more so in the case of a larger and more hydrophobic capsid building block. Interestingly, our unsuccessful attempts to construct an $\mathrm{I}_{6}$ ELP-phage are consistent with these results. In this case, if the growth inhibition is solely due to effects of 220 intracellular I $_{6}$ ELP-pVIII levels, incubation at lower temperatures or in cell-free systems may circumvent this problem.

(a)

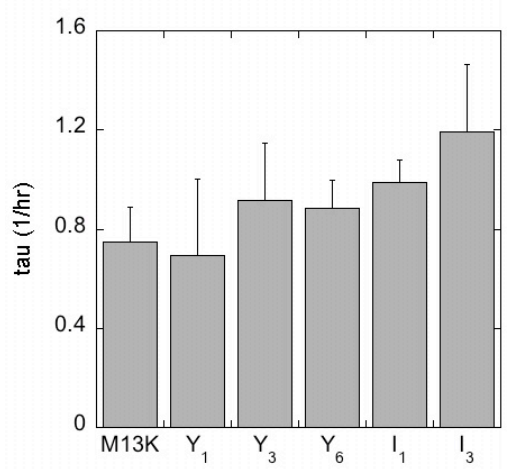

(b)

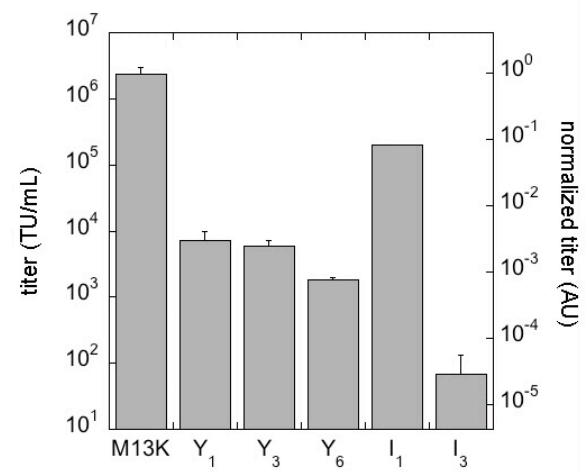

Fig. 2 (a) Time constants for TG1 E.coli bacteria infected with ELP-phage DNA. Note that ELPphage particles are secreted throughout bacterial growth. In general, longer ELP inserts result in 225 slower growth as compared to the parental M13KO7. (b) Infectivity of ELP-phages determined by titering. Here, longer ELP inserts on phages lead to a reduced ability to infect bacteria.

The ability of ELP-phage particles to infect $E$. coli is substantially reduced due to the presence of the ELP, except for $I_{1}$ (Figure $2 b$ ). Such a result is intriguing, given that infectivity is mediated by the minor coat protein (pIII) on the viral capsid

230 binding to the bacterial coat protein pilin and not the pVIII protein where the ELP is displayed. $^{13}$ However, the titering assay cannot discriminate among any of the events involved in infection: phage-bacteria binding, DNA insertion, DNA replication, protein synthesis, and secretion. As seen in Figure 2a, once the phage DNA is internalized, bacterial growth is negatively impacted. Inhibition of any one 235 or several of the remaining steps needed to complete the phage "life cycle" could explain the results in Figure $2 b$. Over the course of several weeks, a gradual decrease in infectivity was also observed (data not shown), indicating a role for nonspecific interactions among phage particles or structural changes in the capsid.

6 | [journal], [year], [vol], 00-00 
Regardless of the mechanism, this would further reduce the availability of phage ${ }_{240}$ particles binding to bacteria, leading to lower overall infectivity.

\section{Dynamic light scattering}

Diffusion of phage particles in dilute solution potentially gives insight into particleparticle aggregation among ELP-phages. To minimize particle-particle effects before reaching the desired transition temperature, all samples were measured at or 245 below a concentration of approximately $\mathrm{c}=1 \times 10^{12}$ virus $/ \mathrm{mL}$ or $30 \mathrm{ug} / \mathrm{mL}$, which is below the overlap ${ }^{30}$ concentration $\mathrm{c}^{*}=1$ particle $/$ length ${ }^{3}=2 \times 10^{12}$ virus $/ \mathrm{mL}$. Due to the rod-like shape of the viral particles, the average translational diffusion coefficent D has unequal contributions from diffusion along the rod axis and the other two orthogonal directions. Nevertheless an "equivalent" hydrodynamic sphere size can

250 be determined and is useful as a first approximation. Above the transition temperature, we initially expected the increased hydrophobicity to drive attractive interactions leading to aggregation and perhaps, precipitation.

Over the temperature range $5-75^{\circ} \mathrm{C}$, the were no significant changes in the $\mathrm{z}$ average size, indicating a lack of particle aggregation. The presentation of the ELP 255 motif on pVIII will increase the diameter of phages, yet surprisingly, $Y_{3}>Y_{1}>Y_{6}$, suggesting that the $\mathrm{Y}_{6}$ ELP-phage may be somewhat collapsed on the capsid surface. Conversely, $\mathrm{Y}_{3}$ may be unusually extended away from the capsid surface. The lack of aggregation can perhaps be best understood in the context of critical solution phenomena, where as recognized by Flory, ${ }^{31}$ low concentrations require that the 260 solvent quality become increasingly "poor" to drive phase separation. Such changes in solvent quality could be achieved by either using more hydrophobic guest residues, such as tryptophan, or by adding chaotropes such as urea.

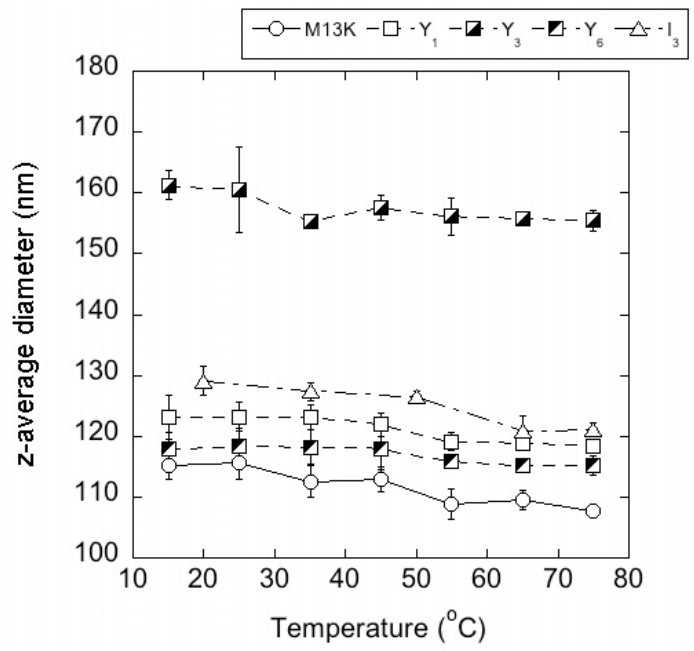

Fig. 3 Average hydrodynamic size of ELP-phages as determined by dynamic light scattering.

\section{Zeta potential}

The major coat protein pVIII possesses acidic residues near its amino terminus and thus the particles are electrostatically stabilized in solution (see Table 1). We surmised that the presence of an ELP insertion $\left(\mathrm{NH}_{2}-\mathrm{AE} \downarrow\right.$ GDD $\left.\ldots\right)$ at or near the

[journal], [year], [vol], 00-00 | 7 
270 terminus would alter the effective charge on the phage capsid. Measurements of the zeta potential at low ionic strength $(1.5 \mathrm{mM} \mathrm{NaCl}, \mathrm{pH} 7)$ showed little to no change in surface charge between ELP-modified particles and the parental M13KO7 phage (Figure 4). This may be due to the ELP motif having predominantly small sidechains, while also being largely ordered and extended below $\mathrm{T}_{\mathrm{t}}$. Presumably above ${ }_{275} T_{t}$ the zeta potential will be reduced by collapse of the ELP chain towards the surface. This effect would be most pronounced for $\mathrm{Y}_{3}$, with the largest apparent hydrodynamic size. At higher $\mathrm{pH}$, as expected, the zeta potential increases for all the tyrosine phages. Here the smallest effect appears to be for $\mathrm{Y}_{6}$, which in view of the DLS results (Figure 3), suggests that the more compact physical conformation of 280 the ELP motif may attenuate the increase surface charge. Of course, the inherent surface charge of the phages provides a barrier to aggregation that is not present for soluble ELP polymers. Site-directed mutagenesis of charged residues in pVIII would directly reveal their role in counteracting aggregation above $T_{t}$.

(a)

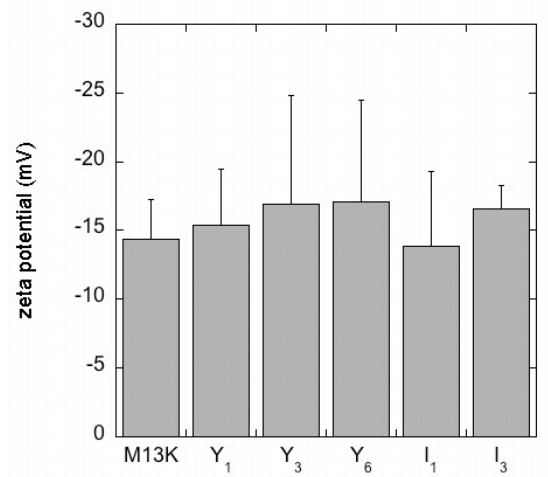

(b)

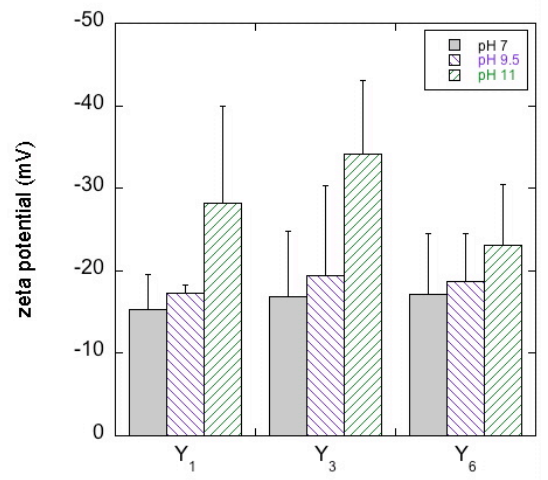

Fig. 4 (a) Zeta potential of ELP-phages in $1.5 \mathrm{mM} \mathrm{NaCl}$ at pH 7, 25 $5^{\circ}$. (b) Dependence of zeta potential on $\mathrm{pH}$ for tyrosine ELP-phages.

\section{Calorimetry}

The absence of micro- and macroscopic aggregation is consistent with the picture of 290 lower critical solution phenomena at dilute concentrations. Nevertheless, the ELP motif can still undergo structural changes to a disordered, compact chain from a open beta-spiral above $T_{t}$. Preliminary calorimetry experiments reveal for both tyrosine and isoleucine ELP-phages, subtle differences in the heating curves can be observed when compared to $\mathrm{M} 13 \mathrm{KO} 7$, between $35-45^{\circ} \mathrm{C}$ (Figure 5). While 295 speculative at this stage, it does appear that shorter tyrosine motifs exhibit higher transition temperatures. The minimum near $75^{\circ} \mathrm{C}$ is an artifact from the range of the heating cycle runs, but in any case protein denaturation of the phage proteins would be likely to occur by this point.

8 | [journal], [year], [vol], 00-00 


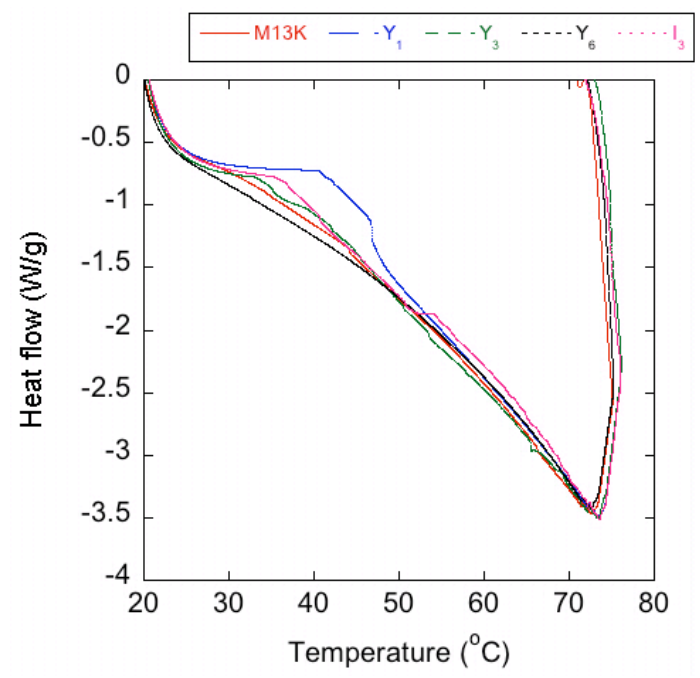

Fig. 5 Differential scanning calorimetry of ELP-phages as compared to the parental M13KO7 phage. Scans were conducted from $20-80^{\circ} \mathrm{C}$ at a rate of $10^{\circ} \mathrm{C} / \mathrm{min}$.

\section{Summary}

The filamentous bacteriophage capsid provides a platform for introducing novel functionality in the form of displayed polypeptides. Stimulus-responsive ${ }_{305}$ polypeptides, such as the ELP motif, are capable of conferring tunable properties to their phage scaffolds. This work is a first attempt to incorporate dynamic control over phage properties such as size and charge - potentially enabling new applications for these materials. In contrast to large ELP polymers that aggregate above their $T_{t}$, it seems clear that much shorter ELP chains grafted to phage particles 310 will remain in solution unless they are sufficiently hydrophobic. However, given that the ELP motif can still switch between an extended to collapsed state implies the ability for capture/release of small molecules.

Further characterization at the protein level using techniques such as circular dichroism should reveal if the ELP motif alters the largely alpha-helical nature of 315 the major coat protein (pVIII). Together with electron/scanning probe microscopies, structural changes in the phage capsid - possibly manifested as changes in preferred curvature or bending stiffness - should also be apparent. Other, more hydrophobic, guest residues such as tryptophan might be capable of inducing aggregation, yet phage production in bacteria may also be more strongly inhibited. Combination of

320 ELP motifs with additional functionality in phage capsids, such as the streptavidinbinding tripeptide HPQ, might allow for the creation of directed phage-based networks with the ELP acting as a stimulus-responsive linker.

\section{Acknowledgements}

The authors thank Sam Pendergraph for initial design contributions to this work and ${ }_{325} \mathrm{H}$. Henning Winter for use of his calorimeter. Support from the Materials Research Science and Engineering Center (MRSEC, DMR-0213695) and the Nanoscale Science and Engineering Center (NSEC, CMMI-0531171) at the University of 
Massachusetts Amherst is gratefully acknowledged.

\section{References}

330 1. K. A. Dill, D. O. Alonso and K. Hutchinson, Biochemistry, 1989, 28, 5439-5449.

2. C. L. Cheung, S. W. Chung, A. Chatterji, T. W. Lin, J. E. Johnson, S. Hok, J. Perkins and J. J. De Yoreo, J Am Chem Soc, 2006, 128, 10801-10807.

3. R. Stoenescu and W. Meier, Chemical Communications, 2002, 3016-3017.

4. Q. Wang, T. Lin, J. E. Johnson and M. G. Finn, Chem Biol, 2002, 9, 813-819.

335 5. Z. Dogic and S. Fraden, Phys Rev Lett, 1997, 78, 2417-2420.

6. F. H. Crick and J. D. Watson, Nature, 1956, 177, 473-475.

7. J. B. Bancroft and P. Kaesberg, Nature, 1958, 181, 720-721.

8. D. Prasuhn, R. M. Yeh, A. Obenaus, M. Manchester and M. G. Finn, Chem Commun (Camb), 2007, 1269-1271.

340 9. P. A. Suci, D. L. Berglund, L. Liepold, S. Brumfield, B. Pitts, W. Davison, L. Oltrogge, K. O. Hoyt, S. Codd, P. S. Stewart, M. Young and T. Douglas, Chem Biol, 2007, 14, 387-398.

10. P. J. Yoo, K. T. Nam, J. Qi, S.-K. Lee, J. Park, A. M. Belcher and P. T. Hammond, Nat Mater, 2006, 5, 234-240.

11. K. T. Nam, B. R. Peelle, S. W. Lee and A. M. Belcher, Nano Lett, 2004, 4, 23-27.

345 12. C. B. Mao, D. J. Solis, B. D. Reiss, S. T. Kottmann, R. Y. Sweeney, A. Hayhurst, G. Georgiou, B. Iverson and A. M. Belcher, Science, 2004, 303, 213-217.

13. D. A. Marvin, Curr Opin Struct Biol, 1998, 8, 150-158.

14. C. M. Soto, A. S. Blum, G. J. Vora, N. Lebedev, C. E. Meador, A. P. Won, A. Chatterji, J. E. Johnson and B. R. Ratna, J Am Chem Soc, 2006, 128, 5184-5189.

350 15. M. Uchida, M. T. Klem, M. Allen, P. Suci, M. Flenniken, E. Gillitzer, Z. Varpness, L. O. Liepold, M. Young and T. Douglas, Advanced Materials, 2007, 19, 1025-1042.

16. E. Gillitzer, P. Suci, M. Young and T. Douglas, Small, 2006, 2, 962-966.

17. Q. Wang, T. Lin, L. Tang, J. E. Johnson and M. G. Finn, Angew Chem Int Ed Engl, 2002, 41, 459-462.

355 18. D. W. Urry, J Phys Chem B, 1997, 101, 11007-11028

19. B. Li, D. O. V. Alonso and V. Daggett, J Mol Biol, 2001, 305, 581-592.

20. C. M. Venkatachalam and D. W. Urry, Macromolecules, 1981, 14, 1225-1229.

21. S. C. Heilshorn, J. C. Liu and D. A. Tirrell, Biomacromolecules, 2005, 6, 318-323.

22. J. Hyun, W.-K. Lee, N. Nath, A. Chilkoti and S. Zauscher, J Am Chem Soc, 2004, 126, 7330360 7335 .

23. D. E. Meyer and A. Chilkoti, Nat Biotechnol, 1999, 17, 1112-1115.

24. Z. Megeed, R. M. Winters and M. L. Yarmush, Biomacromolecules, 2006, 7, 999-1004.

25. H. Reiersen and A. R. Rees, Biochemistry, 1999, 38, 14897-14905.

26. D. E. Meyer and A. Chilkoti, Biomacromolecules, 2004, 5, 846-851.

365 27. H. Bermudez, S. Kontos and J. A. Hubbell, in preparation, 2007.

28. H. Reiersen, A. R. Clarke and A. R. Rees, J Mol Biol, 1998, 283, 255-264.

29. Z. Megeed, R. M. Winters and M. L. Yarmush, Biomacromolecules, 2006, 7, 999-1004.

30. S. Schulz, E. Maier and R. Weber, J Chem Phys, 1989, 90, 7-10.

31. P. J. Flory, J Chem Phys, 1941, 9, 660-661.

370

10 | [journal], [year], [vol], 00-00 